\title{
Simulating single-spin dynamics on an IBM five-qubit chip
}

\author{
Émerson M. Alves ${ }^{1}$, Francisco D. S. Gomes ${ }^{1}$, Hércules S. Santana ${ }^{1}$, Alan C. Santos ${ }^{* 2 @ ~}$ \\ ${ }^{1}$ Universidade Regional do Cariri, Departamento de Física, Juazeiro do Norte, CE, Brasil \\ ${ }^{2}$ Universidade Federal Fluminense, Instituto de Física, Niterói, RJ, Brasil
}

Received on October 22, 2019. Revised on December 24, 2019. Accepted on December 30, 2019.

\begin{abstract}
In this paper we show how the IBM superconducting chips can be a powerful tool for teaching foundations of quantum mechanics for undergraduate students (for graduates as well, in some cases). To this end, we briefly discuss about the main elements of the IBM Quantum Experience platform necessary to understand this paper, i.e., how to implement operations and single-qubit measurements. We experimentally study the dynamics of single spin systems interacting with static and time-dependent magnetic fields. First, we study the resonant behavior of a single spin coupled to a time-dependent rotating magnetic field. To end, we study the Larmor precession phenomenon. In both cases we show the theoretical and real experimental implementation. This article could be useful in introductory courses on quantum mechanics and nuclear magnetic resonance foundations, for example. Keywords: superconducting chips, quantum mechanics, single spin systems, Larmor precession, nuclear magnetic resonance.
\end{abstract}

\section{Introduction}

In traditional courses of quantum mechanics where we introduce the notion of single-spin dynamics, some interesting results are discussed in a theoretical way, without any experimental implementation or verification. On the other hand, simulating quantum physics is an interesting task that we can explore in the IBM Team's quantum plataform, namely, the IBM Quantum Experience (IBM-QE) [1]. From such platform we have access to a five-qubit system [2] in which we can manipulate it from unitary operations (unitary operators) and measurements. This platform has been used to experimentally confirm some theoretical results on quantum information and computation 3 7] as well as to implement high technology quantum protocols like teleportation 8, 9, among others 10 15.

Methods to circumvent experimental limitations of teaching quantum physics have been considered in literature through simulations 16 18, experimental proposal in quantum optics [19], nuclear magnetic systems [20] and classical implementation of quantum computers 21]. By using a different approach, in this paper we discuss how useful the IBM-QE can be in a physics teaching scenario, where we develop a didactic strategy to deal with the problem of experimentally present foundations of singlespin dynamics in presence of a magnetic field, including time-dependent fields. To this end, we first present the relevant elements that we need to know before starting the simulation of the examples we will present here. We discuss about the unitary operators that we will use in our simulations and how we can implement measure-

*Correspondence email address: ac_santos@id.uff.br ments in IBM-QE. By the way, we adopt an approach which allows us to introduce how measurement of some physical quantities are performed in a Nuclear Magnetic Resonance (NMR) experimental setup [22]. Then, we discuss about the first interesting phenomena of single-spin dynamics, namely spin resonance phenomena, associated with a spin- $\frac{1}{2}$ particle in presence of a strong static field along $z$-direction and a rotating magnetic field. We discuss how the resonance phenomenon emerges from a suitable choice of the rotating magnetic field frequency and how it allows us to promote transitions between "spin up" and "spin down" states of the system, even when such rotating field is weakly interacting with the system 22, 23. To conclude, we study the Larmor spin precession behavior.

\section{The IBM Quantum Experience}

The five-qubit IBM quantum chip is composed by five superconducting transmon qubits operating at a temperature scale around $15 \mathrm{mK} 2426$. A superconducting transmon qubit is composed of two coupled Josephson junctions, with Josephson energy $E_{\mathrm{J}}$ and capacitance $C_{\mathrm{J}}$ [27, and shunted by an additional external capacitance $C_{\mathrm{E}}$, as shown in Fig. 1a. As sketched in Fig. 1b. this system presents a quantized energy levels structure $E_{n}$, where two of these energy levels (the two lowestenergy levels) are used as an artificial qubit for quantum computation, since quantum coherence is observed in such systems [28]. Because the Josephson junctions are superconducting devices, the system is composed by a large number of Cooper pairs (a pair of electrons), where the collective effect of these pairs allows us to use a su- 


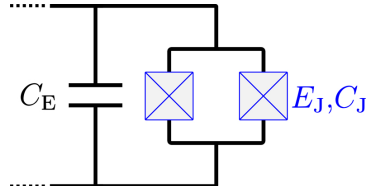

(a)

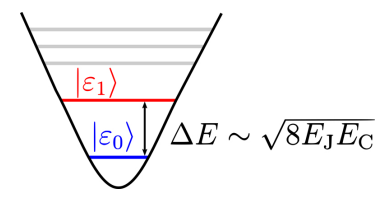

(b)
Figure 1: 1a Sketch of the superconducting circuit that constitutes a transmon superconducting qubit, where we have two Josephson junctions with Josephson energy $E_{\jmath}$ and capacitive energy $E_{\mathrm{C}}$, connected to a external capacitance $C_{\mathrm{E}}$. (1b) The representation (out of scale) of the energy levels of the system, where we highlight the energy levels used as the physical qubit.

perconducting qubit as "artificial atom". In particular, we can use it as a two-level system. For this reason, this system is not a genuine two-level quantum system, like spin- $1 / 2$ particle in presence of a magnetic field or the polarization states of light. However, the characteristics and dynamics of superconducting qubits allows us to simulate such systems. For more relevant details about the superconducting transmon qubits, we recommend the Refs $25,26,28$.

In particular, we can perform simulations and real experimental realizations from two different quantum chips, known as the IBM Q 5 Tenerife (ibmqx4) and IBM Q 5 Yorktown (ibmqx2) quantum chips. Particularly, throughout this article we implement the experiments on IBM Q 5 Yorktown quantum chip, due to the good decoherence time scale of its qubits [1]. In this section we discuss on some important elements of the IBM-QE to be considered in this paper, but more information about IBM-QE quantum chips can be obtained from a number of papers in literature [8, as well as from the own IBM-QE team beginners guide [29].

\subsection{IBM-QE single-qubit operations}

Single qubit operations are unitary transformations on two level systems which, in a realistic physical scenario, arise as result of a dynamics provided by Schrödinger equation

$$
i \hbar|\dot{\psi}(t)\rangle=H(t)|\psi(t)\rangle
$$

for some time dependent (or time independent) Hamiltonian $H(t)$. In particular, single-qubit operations can be obtained from different quantum evolutions, as in spin dynamics 20, 30] or in a quantum particle in a double-well [31], for example. In this paper, the relevant single-qubit operations implementable by the IBM five-qubit chip are

$$
\begin{aligned}
& U_{1}(\lambda)=\left[\begin{array}{cc}
1 & 0 \\
0 & e^{i \lambda}
\end{array}\right], U_{2}(\lambda, \phi)=\left[\begin{array}{cc}
\frac{1}{\sqrt{2}} & -\frac{e^{i \lambda}}{\sqrt{2}} \\
e^{i \phi} & \frac{e^{i(\lambda+\phi)}}{\sqrt{2}}
\end{array}\right], \\
& U_{3}(\theta, \phi, \lambda)=\left[\begin{array}{cc}
\cos \left(\frac{\theta}{2}\right) & -e^{i \lambda} \sin \left(\frac{\theta}{2}\right) \\
e^{i \phi} \sin \left(\frac{\theta}{2}\right) & e^{i(\lambda+\phi)} \cos \left(\frac{\theta}{2}\right)
\end{array}\right],
\end{aligned}
$$

where $\lambda, \phi$ and $\theta$ are free real parameters that can be adjusted in accordance with the gate to be implemented. It is possible to see that the gates $U_{1}(\lambda)$ and $U_{2}(\lambda, \phi)$ can be obtained from gate $U_{3}(\theta, \phi, \lambda)$, but under experimental viewpoint the gates $U_{1}(\lambda)$ and $U_{2}(\lambda, \phi)$ can be more efficient than its counterpart obtained from $U_{3}(\theta, \phi, \lambda)$. In fact, each gate can be characterized from its run-time (time associated with real pulse sequence on transmon qubits), where the run-time for $U_{1}(\lambda)$ can be neglected, $U_{2}(\lambda, \phi)$ is a gate with duration of 1 unit of gate time and $U_{3}(\theta, \phi, \lambda)$ has run-time of 2 units of gate time. Therefore, it is most convenient to use the gates $U_{1}(\lambda)$ and $U_{2}(\lambda, \phi)$ whenever possible.

\subsection{Measuring physical quantities in a single-spin system}

The measurement process in physics depends on the system under consideration $20,30,32,34$. Here, we are interested in studying a single-spin- $\frac{1}{2}$ system driven by magnetic fields which act on it. Thus, since the system can be found in two different orthogonal states $|\uparrow\rangle$ and $|\downarrow\rangle$ associated with different values of the spin component, these states work as a computational basis in a Nuclear Magnetic Resonance based quantum computer [20, 30]. In particular, the IBM-QE allows us to implement measurements in computational basis $|0\rangle \equiv|\uparrow\rangle$ and $|1\rangle \equiv|\downarrow\rangle$, i.e., given a quantum state $|\psi\rangle=a|\uparrow\rangle+b|\downarrow\rangle$, the measurement outcome provides values for $|a|^{2}$ and $|b|^{2}$. Thus, the physical quantities which we can measure in IBM-QE are constrained to a set of measurements that can be obtained from parameters $|a|^{2}$ and $|b|^{2}$. For example, if we want to measure the expected value of the spin along $z$ direction, we have

$$
\mathcal{M}_{z}=\left\langle\psi\left|S_{z}\right| \psi\right\rangle=\frac{\hbar}{2}\left(|a|^{2}-|b|^{2}\right),
$$

where $S_{x}, S_{y}$ and $S_{z}$ are the spin operators defined as

$$
\begin{aligned}
& S_{x}=\frac{\hbar}{2}\left[\begin{array}{ll}
0 & 1 \\
1 & 0
\end{array}\right], \quad S_{y}=\frac{\hbar}{2}\left[\begin{array}{cc}
0 & -i \\
i & 0
\end{array}\right], \\
& \text { and } S_{z}=\frac{\hbar}{2}\left[\begin{array}{cc}
1 & 0 \\
0 & -1
\end{array}\right] .
\end{aligned}
$$

Thus, it is possible to compute $\mathcal{M}_{z}$. In addition, if we want to compute the expected value of spin along $x$ direction, we need to compute $\mathcal{M}_{x}=\left\langle\psi\left|S_{x}\right| \psi\right\rangle$. It can be done if we define the operator

$$
H=\frac{1}{\sqrt{2}}\left[\begin{array}{cc}
1 & 1 \\
1 & -1
\end{array}\right]
$$


where one uses the relation $S_{x}=H S_{z} H$ to write

$$
\mathcal{M}_{x}=\left\langle\psi\left|H S_{z} H\right| \psi\right\rangle=\left\langle\psi_{x}\left|S_{z}\right| \psi_{x}\right\rangle,
$$

with $\left|\psi_{x}\right\rangle=H|\psi\rangle$. In quantum computation, the operator $H$ is a Hadamard gate and it can be implemented on IBM-QE. In conclusion, it means we can measure $\mathcal{M}_{x}$ if we implement a Hadamard gate on the state $|\psi\rangle$ and then we measure the output state in computational basis. In a similar way, the measurement of the quantity $\mathcal{M}_{y}=$ $\left\langle\psi\left|S_{y}\right| \psi\right\rangle$ can be done by defining the rotation operator

$$
R_{x}(\xi)=\left[\begin{array}{cc}
\cos (\xi / 2) & -i \sin (\xi / 2) \\
-i \sin (\xi / 2) & \cos (\xi / 2)
\end{array}\right],
$$

that represents a rotation of an angle $\xi$ around $X$ direction 23. Thus, if we implement the operation with $\xi=-\pi / 2$ we get

$$
R_{x}(-\pi / 2)=\frac{1}{\sqrt{2}}\left[\begin{array}{ll}
1 & i \\
i & 1
\end{array}\right],
$$

so that we can show that $S_{y}=R_{x}(-\pi / 2) S_{z} R_{x}^{\dagger}(-\pi / 2)$, then

$$
\begin{aligned}
& \mathcal{M}_{y}=\left\langle\psi\left|S_{y}\right| \psi\right\rangle=\left\langle\psi\left|R_{x}(-\pi / 2) S_{z} R_{x}^{\dagger}(-\pi / 2)\right| \psi\right\rangle \\
& =\left\langle\psi_{y}\left|S_{z}\right| \psi_{y}\right\rangle
\end{aligned}
$$

with $\left|\psi_{y}\right\rangle=R_{x}^{\dagger}(-\pi / 2)|\psi\rangle$. Thus, we can measure any spin component along the directions $x, y$ and $z$. In addition, we can perform measurement of any physical quantity $\left(\mathbb{D}\right.$ which can be written as $\mathbb{D}=R_{\hat{r}}(\phi) S_{z} R_{\hat{r}}^{\dagger}(\phi)$, where $R_{\hat{r}}(\phi)$ denotes a rotation of an angle $\phi$ around direction $\hat{r}$, from equation

$$
\mathcal{O}=\langle\psi|\mathbb{D}| \psi\rangle=\left\langle\psi\left|R_{\hat{r}}(\phi) S_{z} R_{\hat{r}}^{\dagger}(\phi)\right| \psi\right\rangle
$$

\section{Single-spin dynamics on IBM Quantum Experience}

The spin is an internal degree of freedom of the electron that can be manipulated through external magnetic fields. In particular, for a spin- $\frac{1}{2}$ particle we have two distinct states $|\uparrow\rangle$ and $|\downarrow\rangle$. These two states satisfy the eigenvalue equation

$$
S_{z}|\uparrow\rangle=\frac{\hbar}{2}|\uparrow\rangle \quad \text { and } \quad S_{z}|\downarrow\rangle=-\frac{\hbar}{2}|\downarrow\rangle,
$$

in which $\hbar$ is the reduced Planck's constant and $S_{n}=$ $(\hbar / 2) \sigma_{n}$, where $\sigma_{n}(n=\{x, y, z\})$ denotes the Pauli matrices for a two-level system given by (with $i=\sqrt{-1}$ )

$$
\begin{gathered}
\sigma_{x}=\left[\begin{array}{ll}
0 & 1 \\
1 & 0
\end{array}\right], \quad \sigma_{y}=\left[\begin{array}{cc}
0 & -i \\
i & 0
\end{array}\right] \\
\text { and } \sigma_{z}=\left[\begin{array}{cc}
1 & 0 \\
0 & -1
\end{array}\right],
\end{gathered}
$$

so that the matrix form for the basis $|\uparrow\rangle$ and $|\downarrow\rangle$ reads as $|\uparrow\rangle=\left[\begin{array}{ll}1 & 0\end{array}\right]^{\mathrm{t}}$ and $|\downarrow\rangle=\left[\begin{array}{ll}0 & 1\end{array}\right]^{\mathrm{t}}$, with superscript " $\mathrm{t}$ " denoting the transpose of a matrix.

\subsection{Simulating Nuclear Magnetic Resonance phenomenon}

Let us consider the coupling dynamics of a single spin- $\frac{1}{2}$ with a rotating magnetic field $\vec{B}(t)$ given by

$$
\vec{B}(t)=\vec{B}_{0}+\vec{B}_{\mathrm{rf}}(t)
$$

where $\vec{B}_{0}=B_{0} \hat{z}$ is the field component along the $Z$ direction and $\vec{B}_{\mathrm{rf}}(t)$ is a time-dependent transverse magnetic fields, given by

$$
\vec{B}_{\mathrm{rf}}(t)=B_{\mathrm{rf}}[\cos (\omega t) \hat{x}+\sin (\omega t) \hat{y}]
$$

where $\omega$ is the rotating frequency of the transverse magnetic field, also known as Radio-Frequency (rf) field. Therefore, the Hamiltonian of the system is written as

$$
H(t)=\frac{\hbar \omega_{0}}{2} \sigma_{z}+\frac{\hbar \omega_{\mathrm{rf}}}{2}\left[\cos (\omega t) \sigma_{x}+\sin (\omega t) \sigma_{y}\right]
$$

where $\omega_{0}$ is the Larmor frequency and $\omega_{\mathrm{rf}}$ is the coupling intensity between the spin and the rf-field. The solution of the Schrödinger equation for the system is known and given by (See Appendix A)

$$
|\psi(t)\rangle=\exp \left[-i \frac{\omega}{2} t \sigma_{z}\right] \exp \left[-\frac{i}{\hbar} \tilde{H} t\right]|\psi(0)\rangle,
$$

where

$$
\tilde{H}=\hbar \frac{\omega_{0}-\omega}{2} \sigma_{z}+\hbar \frac{\omega_{\mathrm{rf}}}{2} \sigma_{x}
$$

In matrix form, we write each evolution operator as

$$
\begin{aligned}
& U_{0}(t)=\exp \left[-i \frac{\omega}{2} t \sigma_{z}\right]=e^{-\frac{i \omega t}{2}}\left[\begin{array}{cc}
1 & 0 \\
0 & e^{i \omega t}
\end{array}\right] \\
& U_{x z}(t)=\exp \left[-\frac{i}{\hbar} \tilde{H} t\right]= \\
& {\left[\begin{array}{cc}
\cos \left(\frac{\Omega t}{2}\right)+i \frac{\Delta}{\Omega} \sin \left(\frac{\Omega t}{2}\right) & i \frac{\omega_{\mathrm{rf}}}{\Omega} \sin \left(\frac{\Omega t}{2}\right) \\
i \frac{\omega_{\mathrm{rf}}}{\Omega} \sin \left(\frac{\Omega t}{2}\right) & \cos \left(\frac{\Omega t}{2}\right)-i \frac{\Delta}{\Omega} \sin \left(\frac{\Omega t}{2}\right)
\end{array}\right],}
\end{aligned}
$$

where $\Delta=\omega_{0}-\omega$ is a detuning between the rf-field and the Larmor frequencies, and $\Omega^{2}=\Delta^{2}+\omega_{\text {rf }}^{2}$ is the effective Rabi frequency, which takes into account the effects due to the detuning [35]. It is worth to mention that the term $e^{-\frac{i \omega t}{2}}$ in $U_{0}(t)$ represents a global phase and can be neglected, as we will do from now on. In particular, here we will simulate the resonance phenomenon, where a small rf-field $\left(\left|\omega_{\text {rf }}\right| \ll\left|\omega_{0}\right|\right)$ can be used to flip the spin state when the rf-field oscillation frequency is close to $\omega_{0}$ $(|\Delta| \rightarrow 0)$. Under this configuration, the operator $U_{x z}(t)$ becomes

$$
U_{x z}^{\mathrm{ress}}(t)=\left[\begin{array}{cc}
\cos \left(\frac{\omega_{\mathrm{rf}} t}{2}\right) & i \sin \left(\frac{\omega_{\mathrm{rf}} t}{2}\right) \\
i \sin \left(\frac{\omega_{\mathrm{rf}} t}{2}\right) & \cos \left(\frac{\omega_{\mathrm{rf}} t}{2}\right)
\end{array}\right],
$$

and the system will evolve as

$$
\left|\psi^{\mathrm{ress}}(t)\right\rangle=U_{0}(t) U_{x z}^{\mathrm{ress}}(t)|\psi(0)\rangle .
$$


In case where the initial state is given by $|\psi(0)\rangle=|\uparrow\rangle$, we compute the time-dependence of the $Z$-spin component and we find

$$
\mathcal{M}_{z}(t)=\left\langle\psi(t)\left|S_{z}\right| \psi(t)\right\rangle=\frac{\hbar}{2} \cos \left(\omega_{\mathrm{rf}} t\right) .
$$

Notice that the parameter $\omega$ does not develop any role in the above quantity. We choose this particular state because the standard input state in IBM quantum chip is the computational state $|0\rangle$, namely the spin-up state $|\uparrow\rangle$.

To simulate the spin dynamics of the Eq. 21) we need to map the evolution operators $U_{0}(t)$ and $U_{x z}^{\text {ress }}(t)$ into the available parameters in IBM-QE. We mean, from the Eqs. (2) we need to identify the operator $U_{0}(t)$ as $U_{1}\left(\lambda_{1}\right)$ and the operator $\mathcal{U}_{\text {ress }}(t)$ as $U_{3}(\theta, \phi, \lambda)$ for a particular choice of the parameters $\lambda, \phi$ and $\theta$. A first point to be highlighted is that $\lambda, \phi$ and $\theta$ are dimensionless parameters, so we need to get a correspondence between the dimensionless parameters of $U_{0}(t)$ and $U_{x z}^{\text {ress }}(t)$ with the parameters associated to the operators $U_{1}\left(\lambda_{1}\right)$ and $U_{3}(\theta, \phi, \lambda)$, respectively. In our case, it is possible to see that $U_{1}(\omega t)=U_{0}(t)$ and $U_{3}\left(\frac{\omega_{\mathrm{rf}} t}{2}, \frac{\pi}{2}, \frac{3 \pi}{2}\right)=\mathcal{U}_{\text {ress }}(t)$. In conclusion, $\mathcal{U}_{\text {ress }}(t)$ would be simulated through the sequence

$$
\mathcal{U}_{\text {ress }}^{\text {sim }}(t)=U_{1}(\omega t) U_{3}\left(\frac{\omega_{\mathrm{rf}} t}{2}, \frac{\pi}{2}, \frac{3 \pi}{2}\right),
$$

The quantum circuit that executes this task is shown in Fig. 2 In Fig. 2a we present the theoretical and experimental data of the spin dynamics for the case where $\omega=\omega_{0}$ (resonance situation). To this end, we encode our spin (to be driven by Hamiltonian in Eq. (15)) as the qubit $q[2]$ shown in Fig. $2 \mathrm{~b}$. The experimental chip condition is shown in table 1 , as provided by IBM team before we start the experimental procedure. In Table 1. we present the frequency $\nu$ of the energy gap between states $|\downarrow\rangle$ and $|\uparrow\rangle$ of the two-level system used in experiment, namely, $\nu=(\Delta E / \hbar)$, where $\Delta E$ is the difference of energy between excited state and fundamental one. In addition, it is shown the relevant quantities $T_{1}$ and $T_{2}$ associated with the natural undesired (decoherence) effects acting on the system (for more details about them, see Sec. B1 fo the Appendix B). Moreover, we present the gate and readout errors in the table. These undesired effects are different from the decoherence ones, in particular the gate error arises from the imprecise control of the

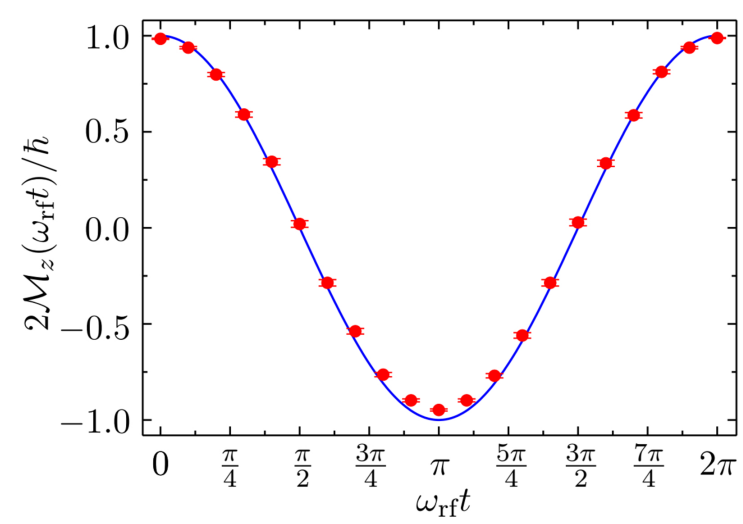

(a)

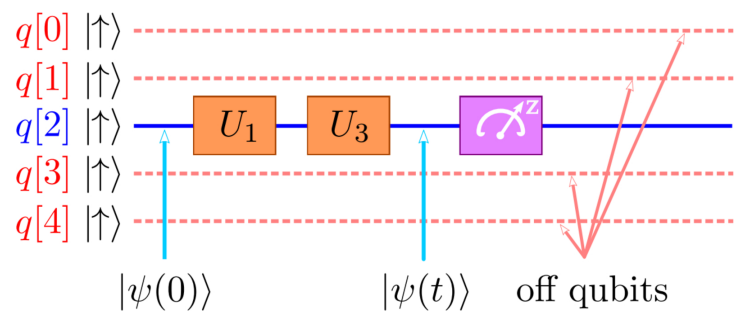

(b)

Figure 2: 2a Theoretical (line) and experimental data (dots) of the single spin measurement at resonance (as multiple of $\hbar / 2$ ). In our experiment we set $N=N_{\max }=8192$ shots. 2b Circuit implemented on IBM Q 5 Yorktown chip (ibmqx2), where we highlight the qubit used in experiment (continuum blue line) while the rest of the qubit were disregarded during the experiment (dashed light red lines). The magenta box represents a measurement in computational basis.

pulses used to manipulate the qubit, while readout error takes into account systematic errors and experimental limitations of the evaluation process to measure the spin components of the qubit. A brief discussion on gate and readout errors is considered in Sec. 2 of the Appendix B.

After implementing the unitary operations (which simulate the dynamics), we measure the $Z$-spin component in order to see some spin flip in the system (as expected in resonance situation). Up to a small experimental error, the experimental results show a good compatibility with the theoretical expected values. The error bar is computed from relation $36 . \Delta p=\pi \sqrt{p_{0}\left(1-p_{0}\right) / N}$, where $N$ is the number of experimental shots and $p_{0}=|\langle\uparrow \mid \psi\rangle|^{2}$. It is important to justify here the choice of the qubit $q[2]$ as our working system. As discussed in Appendix B, the

Table 1: Physical parameters obtained from last calibration before the experimental implementation shown in Fig 2 . For this experiment, the calibration date is 2019-05-24 08:04:12 AM. See Appendix B for more details about the physical meaning of each quantity in this table.

\begin{tabular}{r|ccccc} 
Parameters & $q[0]$ & $q[1]$ & $q[2]$ & $q[3]$ & $q[4]$ \\
\hline Frequency $(\mathrm{GHz})$ & 5.29 & 5.24 & 5.03 & 5.3 & 5.08 \\
$\mathrm{~T} 1(\mu \mathrm{s})$ & 53.40 & 62.10 & 65.50 & 60.90 & 49.50 \\
$\mathrm{~T} 2(\mu \mathrm{s})$ & 42.20 & 54.40 & 57.20 & 28.90 & 59.90 \\
Gate error $\left(10^{-3}\right)$ & 3.35 & 1.55 & 4.64 & 3.44 & 5.84 \\
Readout error $\left(10^{-2}\right)$ & 4.80 & 24.20 & 1.70 & 1.70 & 32.00
\end{tabular}


scale $T_{1}$ and $T_{2}$ times shown in Table 1 are related with the timelife of the coherence in system, so the quantum gates should be implemented during a computation run time $T_{\text {comp }} \ll T_{1}$ and $T_{\text {comp }} \ll T_{2}$. Typically, the time required to implement each gate is of the order of $10 \mathrm{~ns}$, so the circuits used in this manuscript are implemented within a computation run time of the order of few tens of nanoseconds 37, 38. Consequently, in our particular experiment we do not need to worry with the decohering effects acting on the system. On the other hand, the gate and readout errors are important for our discussion. In order to minimize these errors in our experiment, we used the physical qubit $q[2]$ because it has the best (smallest) gate and readout errors. Eventually, one obtains a different result if one chooses another option. In particular, if we choose the physical qubit $q[4]$, it is not expected a good compatibility of our results with the theoretical expected values.

\subsection{Spin precession}

Now, as a second application, we discuss the experimental simulation of the Larmor precession phenomenon. Let us consider the system at the initial state

$$
\left|\psi_{0}\right\rangle=\cos \left(\frac{\theta}{2}\right)|\uparrow\rangle+\sin \left(\frac{\theta}{2}\right)|\downarrow\rangle
$$

where $\theta \in[0, \pi]$. If the system is driven by a timeindependent magnetic field along $z$-direction, i.e., $\vec{B}_{0}=$ $B_{0} \hat{z}$, the associated Hamiltonian is read as

$$
H_{0}=\frac{\hbar \omega_{0}}{2} \sigma_{z}
$$

the evolved state of the system will be given by (up to a global phase)

$$
|\psi(t)\rangle=\tilde{U}_{0}(t)\left|\psi_{0}\right\rangle=\cos \left(\frac{\theta}{2}\right)|\uparrow\rangle+e^{i \omega t} \sin \left(\frac{\theta}{2}\right)|\downarrow\rangle,
$$

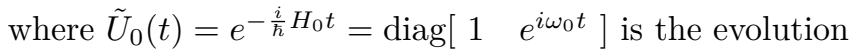
operator. Now, by computing the behavior of the physical quantities defined in Eqs. (3), (6) and (9), we get

$$
\begin{aligned}
& \mathcal{M}_{z}=\frac{\hbar}{2} \cos (\theta), \quad \mathcal{M}_{x}(t)=\frac{\hbar}{2} \sin (\theta) \cos \left(\omega_{0} t\right), \\
& \mathcal{M}_{y}(t)=\frac{\hbar}{2} \sin (\theta) \sin \left(\omega_{0} t\right) .
\end{aligned}
$$

In order to see the precession behavior, if we define the spin vector $\overrightarrow{\mathcal{M}}_{x y}$ in $x y$-plane as

$$
\overrightarrow{\mathcal{M}}_{x y}=\mathcal{M}_{x} \hat{x}+\mathcal{M}_{y} \hat{y}
$$

so that we use the Eqs. 27) and we get

$$
\overrightarrow{\mathcal{M}}_{x y}=\frac{\hbar}{2} \sin (\theta)\left[\cos \left(\omega_{0} t\right) \hat{x}+\sin \left(\omega_{0} t\right) \hat{y}\right]
$$

The geometrical representation of $\overrightarrow{\mathcal{M}}_{x y}$ is a spinning vector around $z$-axis (in the $x y$-plane) and its norm is $\left\|\overrightarrow{\mathcal{M}}_{x y}\right\|=\hbar|\sin (\theta)| / 2$. Therefore, when we define the spin vector $\overrightarrow{\mathcal{M}}=\mathcal{M}_{z} \hat{z}+\overrightarrow{\mathcal{M}}_{x y}$ we get

$$
\overrightarrow{\mathcal{M}}(t)=\frac{\hbar}{2} \cos (\theta) \hat{z}+\frac{\hbar}{2} \sin (\theta)\left[\cos \left(\omega_{0} t\right) \hat{x}+\sin \left(\omega_{0} t\right) \hat{y}\right]
$$

since $\mathcal{M}_{z}=\hbar \cos (\theta) / 2$. The geometrical representation of $\overrightarrow{\mathcal{M}}(t)$ is shown in Fig. 3a. For this reason, we call such dynamics Larmor spin precession or spin precession.

To simulate such dynamics, we need to prepare the initial input state $\left|\psi_{0}\right\rangle$, then we implement the evolution as provided by the evolution operator $\tilde{U}_{0}(t)$. Then, we measure the spin component along directions $x, y$ and $z$. Thus, the circuit which simulates the spin precession is shown in Fig. 3 and we can build it following three steps:

(1) The initial state: As mentioned, the IBM-QE has a natural input state given by $|0\rangle=|\uparrow\rangle$, so any algorithm input state should be achieved from it. In particular, the initial state $\left|\psi_{0}\right\rangle$ can be obtained from $|\uparrow\rangle$ through the unitary operation

$$
U_{\text {inp }}=\left[\begin{array}{cc}
\cos \left(\frac{\theta}{2}\right) & -\sin \left(\frac{\theta}{2}\right) \\
\sin \left(\frac{\theta}{2}\right) & \cos \left(\frac{\theta}{2}\right)
\end{array}\right]
$$

The above unitary operator is obtained from the gate $U_{3}(\theta, \phi, \lambda)$ in Eq. (2) if we set $\lambda=0 \phi=0$. Thus, we have $U_{\text {inp }}=U_{3}(\theta, 0,0)$.

(2) The evolution: As previously discussed, the evolution as provided by operator $\tilde{U}_{0}(t)$ can be achieved through the $U_{1}(\lambda)$ gate (up to a global phase) whenever we set $\lambda=\omega_{0} t$.

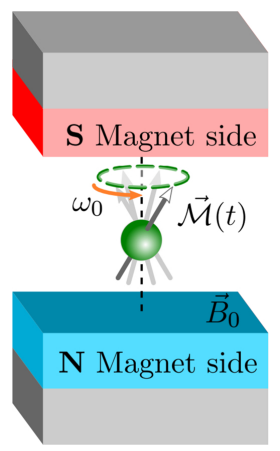

(a)

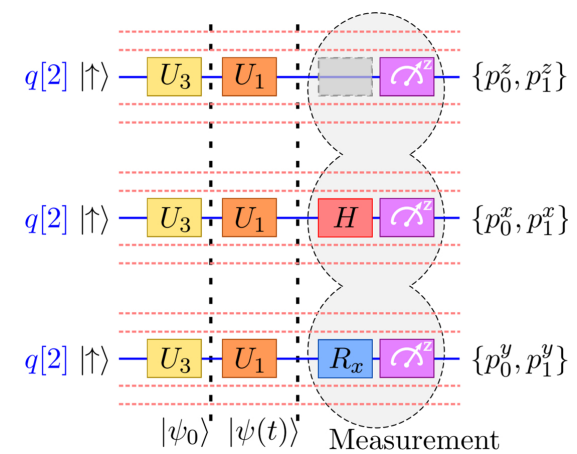

(b)
Figure 3: 3a Sketch of a single-spin inside a static magnetic field along $Z$-direction, where the precession behavior is highlighted. (3b) Circuit used for each dynamics and measurement implemented on IBM Q 5 Yorktown chip (ibmqx2), where we highlight the qubit used in experiment (continuum blue line) while the rest of the qubits are disregarded during the experiment (dashed light red lines). The magenta box represents a measurement in computational basis and provides us the probabilities $\left\{p_{0}^{x, y, z}, p_{1}^{x, y, z}\right\}$. 
(3) The measurement: The last circuit step is the measurement. To measure the $z$-spin component we do not need to implement rotations, since the natural measurement basis of the IBM-EQ is the computational basis ("spin-up " and "spin-down" basis in our case). The spin measurement along $x$ and $y$ direction are obtained as discussed in Sec. 2.2, so we need to implement a Hadamard $(H)$ gate and $R_{x}=R_{x}^{\dagger}(-\pi / 2)$ gate to measure in $x$ and $y$ basis, respectively.

The experiment of the precession spin was implemented with same qubit as in previous experiment, but with new physical parameters as shown in Table 2 In experiment we have two dimensionless parameters to be adjusted, namely the parameter $\theta$ associated with initial state and the quantity $\omega_{0} t$ due to the time-dependence of the operator $\tilde{U}_{0}(t)$. Thus, we choose two values of $\theta$ and we experimentally study the behavior of the physical quantities defined in Eq. (27) as function of $\omega_{0} t$. Since we need to start the protocol with the same state for each measurement, we set the same parameter $\theta$ in gate $U_{3}$ for each circuit of the Fig. 3b while we vary the parameter $\lambda$ of the gate $U_{1}$ used to encode the dimensionless value of $\omega_{0} t$. Given a same initial for all experiments, each circuits in figures provides us the probabilities values $p_{0}^{x, y, z}$ and $p_{1}^{x, y, z}$, then we obtain a set of experimental values of the quantities $\mathcal{M}_{x, y, z}^{\exp }\left(\omega_{0} t\right)$ from equation

$$
\mathcal{M}_{x, y, z}^{\exp }\left(\omega_{0} t\right)=\frac{\hbar}{2}\left[p_{0}^{x, y, z}\left(\omega_{0} t\right)-p_{1}^{x, y, z}\left(\omega_{0} t\right)\right]
$$

Therefore, it allows us to compare the experimental result with theoretical values given in Eq. (27) and the results are shown in Fig. 4 . Again, we compute the error bar for each circuit from relation [36 $\Delta p^{x, y, z}=$ $\pi \sqrt{p_{0}^{x, y, z}\left(1-p_{0}^{x, y, z}\right) / N}$. Thus, we can see the good agreement between theoretical predictions and experimental data.

\section{Conclusion}

Here we have presented a didactic proposal which can be used to present a more consistent study on quantum single-spin dynamics, where we discussed how to remotely implement its experimental realization in a superconducting quantum processor. To this end, we encourage the use of the IBM-QE platform as a resource

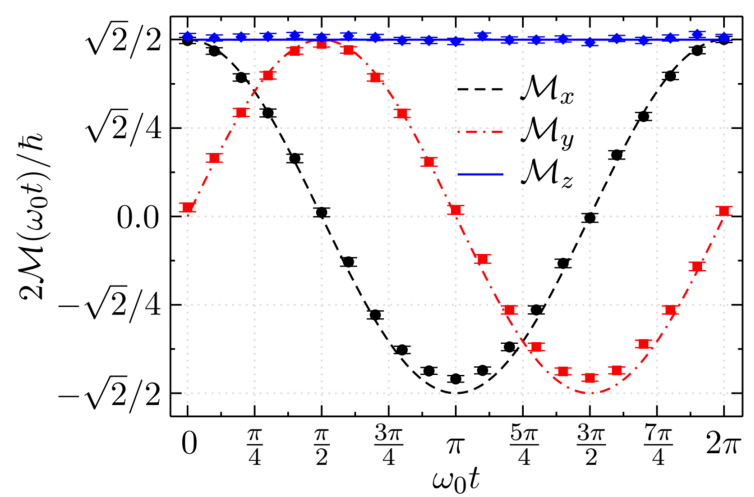

(a)

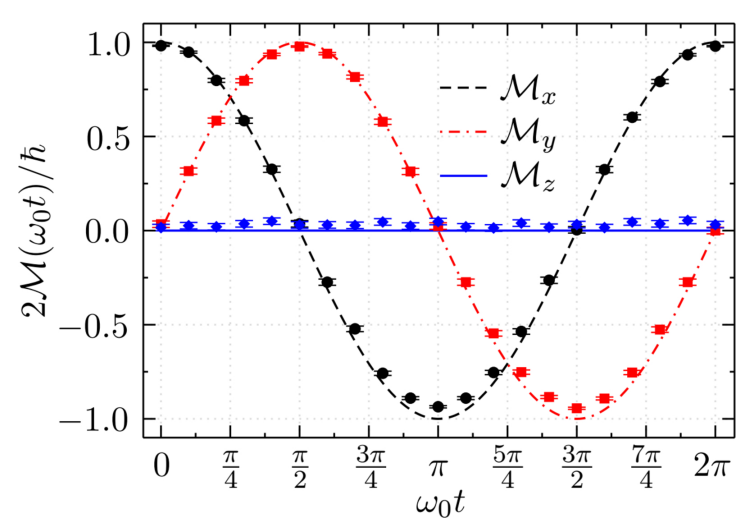

(b)

Figure 4: Spin measurement $\mathcal{M}$ (in multiple of $\hbar / 2$ ) along the direction $x$ (dashed black curves), $y$ (dashed red dot curves) and $z$ (continuum blue curves) as function of $\omega_{0} t$ for the choices (4a) $\theta=\pi / 4$ and (4b) $\theta=\pi / 2$. In our experiment we set $N=N_{\max }=8192$ shots and we implement the circuit on IBM Q 5 Yorktown chip (ibmqx2). Points denote the experimental data obtained from IBM-QE platform.

for teaching quantum mechanics from an experimental approach, in completeness with the theoretical one. Here we have shown the required elements (quantum gates and measurement) to study single-spin dynamics from IBM-QE systems. As a demonstration of how useful the IBM-QE platform can be, we discuss about two particular and interesting dynamics of single-spin system. As a first example, we have discussed about the resonance phenomena in systems composed of nuclear spins, with high applicability in nuclear magnetic resonance based quantum technologies. To conclude, we have studied the

Table 2: Physical parameters obtained from last calibration before the experimental implementation shown in Fig 4 . For this experiment, the last calibration date is 2019-05-29 08:07:25 AM. See Appendix B for more details about the physical meaning of each quantity in this table.

\begin{tabular}{r|ccccc} 
Parameters & $q[0]$ & $q[1]$ & $q[2]$ & $q[3]$ & $q[4]$ \\
\hline Frequency $(\mathrm{GHz})$ & 5.29 & 5.24 & 5.03 & 5.3 & 5.08 \\
$\mathrm{~T} 1(\mu \mathrm{s})$ & 21.90 & 51.10 & 70.10 & 60.30 & 50.40 \\
$\mathrm{~T} 2(\mu \mathrm{s})$ & 26.50 & 42.10 & 62.20 & 27.10 & 51.20 \\
Gate error $\left(10^{-3}\right)$ & 9.28 & 1.46 & 4.21 & 3.95 & 3.44 \\
Readout error $\left(10^{-2}\right)$ & 9.50 & 26.10 & 1.60 & 3.70 & 37.90
\end{tabular}


Larmor precession phenomena. Both applications could be experimentally verified. It is worth to highlight that both experiments were implemented in a relatively short time, for our case, in average, it took between 3 and 10 minutes for getting the experimental data file for each execution (each point of the graph).

The single-qubit dynamics studied in this paper can be implementable through different physical systems. For example, by using the photon polarization, a number of half-wave plate and quarter-wave plate can be used to simulate the dynamics. In this system, the readout process can be done through state tomography of the photon polarization. However, because this system needs a laboratory with high technology electronic devices, such laboratories are not accessible for a large number of universities. A second possibility of physical system, which can implement the experiment discussed here can be, is a NMR experimental setup. However, these system are orders of magnitude more expensive than optical ones. Therefore, the IBM-QE project is a possibility of experimental realizations on quantum information and computation for a number of universities around the world.

\section{Acknowledgments}

ACS acknowledges financial support from the Brazilian agencies Conselho Nacional de Desenvolvimento Científico e Tecnológico (CNPq), Brazilian National Institute of Science and Technology for Quantum Information $[\mathrm{CNPq}$ INCT-IQ (465469/2014-0)] and the Coordenação de Aperfeiçoamento de Pessoal de Nível Superior (CAPES) (Finance Code 001). We are extremely grateful to IBM-QE project and to IBM-QE team for providing the experimental data used in this manuscript. To end, it is important to mention that the results and discussions expressed in this manuscript are the viewpoint of the authors and do not reflect the official policy or position of IBM or the IBM-QE team.

\section{Supplementary material}

The following online material is available for this article: Appendix A: Exact solution for spin- $\frac{1}{2}$ dynamics in a rotating magnetic field.

Appendix B: Non-unitary effects in superconducting transmon qubits.

\section{References}

[1] https://quantum-computing.ibm.com/composer.

[2] B. Schumacher, Phys. Rev. A 51, 2738 (1995).

[3] M. Berta, S. Wehner and M.M. Wilde, New J. Phys. 18, 073004 (2016).

[4] D. García-Martín and G. Sierra, Journal of Applied Mathematics and Physics 6, 1460 (2018).
[5] R.P. Rundle, P.W. Mills, T.Tilma, J.H. Samson and M.J. Everitt, Phys. Rev. A 96, 022117 (2017).

[6] G.W. Dueck, A. Pathak, M. Mazder Rahman, A. Shukla and A. Banerjee, arXiv:1810.00129 (2018).

[7] K. Das and A. Sadhu, arXiv:1810.03390 (2018).

[8] A.C. Santos, Rev. Bras. Ensino Fís. 39, e1301 (2017).

[9] S. Fedortchenko, arXiv:1607.02398 (2016).

[10] N.N. Hegade, A. Das, S. Seth and P.K. Panigrahi, arXiv:1904.12187 (2019).

[11] S. Mahanti, S. Das, B.K. Behera and P.K. Panigrahi, Quantum Inf. Process. 18, 219 (2019).

[12] S.I. Doronin, E.B. Fel'dman and A.I. Zenchuk, Quantum Inf. Process. 19, 68 (2020).

[13] S.J. Devitt, Phys. Rev. A 94, 032329 (2016).

[14] A. Zhukov, S. Remizov, W. Pogosov and Y.E. Lozovik, Quantum Inf. Process. 17, 223 (2018).

[15] O. Viyuela, A. Rivas, S. Gasparinetti, A. Wallraff, S. Filipp and M.A. Martín-Delgado, arXiv:1607.08778 (2018).

[16] J.R. Merrill, Am. J. Phys. 40, 138 (1972).

[17] G. Benenti and G. Strini, Am. J. Phys. 76, 657 (2008).

[18] J.P. Grivet, Am. J. Phys. 61, 1133 (1993).

[19] J. Skaar, J.C. García Escartín and H. Landro, Am. J. Phys. 72, 1385 (2004).

[20] T.F. Havel, D.G. Cory, S. Lloyd, N. Boulant, E.M. Fortunato, M.A. Pravia, G. Teklemariam, Y.S. Weinstein, A. Bhattacharyya and J. Hou, Am. J. Phys. 70, 345 (2002).

[21] Y. Vianna, M.R. Barros and M. Hor-Meyll, Am. J. Phys. 86, 914 (2018).

[22] I. Oliveira, R. Sarthour Jr, T. Bonagamba, E. Azevedo and J.C. Freitas, NMR quantum information processing (Elsevier, Oxford, 2011).

[23] M.A. Nielsen and I.L. Chuang, Quantum Computation and Quantum Information: 10th Anniversary Edition (Cambridge University Press, New York, 2011), 10th ed.

[24] https://www.research.ibm.com/ibm-q/learn/what-isquantum-computing/.

[25] J. Koch, T.M. Yu, J. Gambetta, A.A. Houck, D.I. Schuster, J. Majer, A. Blais, M.H. Devoret, S.M. Girvin and R.J. Schoelkopf, Phys. Rev. A 76, 042319 (2007).

[26] J.A. Schreier, A.A. Houck, J. Koch, D.I. Schuster, B.R. Johnson, J.M. Chow, J.M. Gambetta, J. Majer, L. Frunzio, M.H. Devoret et al., Phys. Rev. B 77, 180502 (2008).

[27] Y. Makhlin, G. Schön and A. Shnirman, Rev. Mod. Phys. 73, 357 (2001).

[28] H. Paik, D.I. Schuster, L.S. Bishop, G. Kirchmair, G. Catelani, A.P. Sears, B.R. Johnson, M.J. Reagor, L. Frunzio, L.I. Glazman et al., Phys. Rev. Lett. 107, 240501 (2011).

[29] https://quantum-computing.ibm.com/supportl.

[30] V. Scarani, Am. J. Phys. 66, 956 (1998).

[31] C. Foot and M. Shotter, Am. J. Phys. 79, 762 (2011).

[32] M.A. Millhouse and D.C. Latimer, Am. J. Phys. 81, 646 (2013).

[33] C. Gerry and P. Knight, Am. J. Phys. 65, 964 (1997).

[34] B. Barman and A. Petrou, Am. J. Phys. 87, 275 (2019).

[35] C.K. Hu, J.M. Cui, A.C. Santos, Y.F. Huang, M.S. Sarandy, C.F. Li and G.C. Guo, Opt. Lett. 43, 3136 (2018).

[36] https://quantumexperience.ng.bluemix.net/proxy/tutorial.

[37] G. Wendin, Reports on Progress in Physics 80, 106001 (2017) 
[38] R. Barends, J. Kelly, A. Megrant, A. Veitia, D. Sank, E. Jeffrey, T.C. White, J. Mutus, A.G. Fowler, B. Campbell et al., Nature 508, 500 (2014). 